\title{
Indirect X-ray detectors based on inkjet-printed photodetectors with a screen-printed scintillator layer
}

Juliana Oliveira, ${ }^{a, b, *}$, Vitor Correia ${ }^{a, b}$, Enrico Sowade $^{c}$, Ikerne Etxebarria ${ }^{d}$, Raul D. Rodriguez, $z^{e}$ Kalyan Y. Mitra ${ }^{c}$, Reinhard R. Baumann ${ }^{c, f}$, Senentxu Lanceros-Mendez, ${ }^{a, d, g, *}$

${ }^{a}$ Universidade do Minho, Centro de Física, 4710-057, Braga, Portugal

${ }^{\mathrm{b}}$ Universidade do Minho, Algoritmi Research Center, Campus de Azurém, 4800-058 Guimarães, Portugal

${ }^{\mathrm{c}}$ Technische Universität Chemnitz, Digital Printing and Imaging Technology, 09107 Chemnitz, Germany

${ }^{\mathrm{d} B C M a t e r i a l s, ~ B a s q u e ~ C e n t e r ~ f o r ~ M a t e r i a l s, ~ A p p l i c a t i o n s ~ a n d ~ N a n o s t r u c t u r e s, ~ U P V / E H U ~}$ Science Park, 48940 Leioa

'Technische Universität Chemnitz, Semiconductor Physics, 09107 Chemnitz, Germany ${ }^{\mathrm{f}}$ Fraunhofer Institute for Electronic Nano Systems (ENAS), Department Printed Functionalities, 09126, Chemnitz, Germany

'IKERBASQUE, Basque Foundation for Science, 48013 Bilbao, Spain

ABSTRACT: Organic photodetectors based on printing technologies will allow to expand the current field of photodetector applications towards large-area and flexible applications in areas such as medical imaging, security and quality control, among others. Inkjet printing is a powerful digital tool for the deposition of smart and functional materials on various substrates, allowing the development of electronic devices such as photodetectors on various substrates. In this work, inkjet-printed photodetector array, based on organic thin-film transistor (OTFT) architecture, have been developed and applied for the indirect detection of X-ray radiation using a scintillator ink as X-ray absorber.

The over $90 \%$ increase of the photocurrent of the photodetectors under X-ray radiation, from about $53 \mathrm{nA}$ without scintillator film to about $102 \mathrm{nA}$ with the scintillator located on 
top of the photodetector, proves the suitability of the developed printed device for X-ray detection applications.

Keywords: inkjet, organic photodetectors, printed photodetectors, radiation detectors, Xray imaging

\section{Introduction}

Photodetectors allow the conversion of light into measurable current signals, being a common component of optoelectronic systems. Photodetectors are thus found in many applications such as automated gates and doors, digital versatile discs (DVD), blue-ray devices, night vision systems, and remote controls ${ }^{1-2}$. Further, they are also implemented in radiation medical imaging ${ }^{3}$, including systems for radiography ${ }^{4}$, mammography ${ }^{5}$, computed tomography $(\mathrm{CT})^{6}$, spectroscopy devices and integrated sensors for lab-on-achip applications ${ }^{7}$. Miniaturization, large-area applicability, selectivity, and high responsivity are key factors that need to be take into account for new developments in this field $^{8-9}$.

The materials typically used for X-ray radiation detection, i.e., conversion of the ionizing radiation into an electrical signal, are mainly based on charge-coupled device (CCD) and complementary metal-oxide-semiconductor (CMOS) technology ${ }^{2}$. However, these methods show limitations regarding the processing into pixelated photodetector matrices applied to large and non-flat areas ${ }^{10}$. The most common material used for this type of radiation photodetector is silicon ${ }^{11}$. Silicon shows limitations related to the low radiation absorption coefficient ${ }^{12}$, which results in the need of high thickness silicon layers in order to obtain high performing devices ${ }^{11,13}$. Nano-structuring of silicon layers can be used to improve the photon absorption, but this approach results in a remarkable cost increase which limits the commercial implementation ${ }^{14}$. 
Thus, there has been an increasing interest on the development of alternative materials for detecting radiation. In particular, efforts are being directed to the development of a new generation of photodetectors for radiation detection, combining easy manufacturing, flexible and seamless integration capabilities and mechanical flexibility ${ }^{15}$. Besides that, several applications, ranging from security control to industrial and medical diagnostics ${ }^{16}$, require thin, conformable sensor panels, with a lower exposure to radiation dose of X-ray imaging systems and for large-area ${ }^{17}$. Printed photodetectors based on organic materials are well-qualified candidates to fulfil the above-mentioned requirements.

All recent investigations related with printed photodetectors have been directly connected with the improvement of light sensitivity yield, the compatibility with silicon and flexible electronics for integration, and should be adapted to low-temperature solution processing ${ }^{18}$. Printed photodetectors show significant advantages in comparison to solid-state devices. They can have large active areas, can be processed at low temperatures, offer an welladjustable spectral sensitivity, and they are inexpensive and easily fabricated ${ }^{13}$. Printing technologies are additive processes that allow a patterned material deposition, the economizing of material usage and the manufacturing at competitive costs ${ }^{19}$.

Among the different printing techniques, inkjet printing ${ }^{20-22}$ is a fully digital technology where the print image is formed by the ejection of single droplets on pre-defined positions of a substrate. The droplets are directly transferred to the substrate without the need for masks which facilitates varying pattern design and customization.

Printed photodetectors are being produced by e.g., inkjet ${ }^{23}$, spraying ${ }^{24-25}$ and screen printing ${ }^{26}$ based on materials such as polymers, silver nanoparticles, carbon nanotubes ${ }^{24}$, metal oxide and even colloidal quantum dots. The combination of low-cost fabrication methods such as printing technologies, represent key advantages of organic semiconductor photodetectors over their silicon-based counterparts ${ }^{23}$. 
The deposition of multilayer stacks of different functional materials provides a simple form of depositing thick films (some nanometers) layer-by-layer onto a surface of any kind or any shape. Multilayers stacks allow to fabricate a sequence of layers, each layer with specific structure and properties, being a rather general approach for the fabrication of devices. This approach has been successfully applied for the development of multilayer ink-jet printed passive components ${ }^{27}$.

In layer-by-layer deposition, the precise structure of each layer depends on a precise set of deposition parameter, which will determine the structure and properties of each layer, but that will allow high level of reproducibility, if the processing parameters are maintained strictly constant ${ }^{27-28}$.

Several concepts have been proposed to promote the advantages of organic semiconductors in medical flat-panel X-ray photodetectors, such as easy processing, flexibility and the possibility of large area fabrication with a low effective cost ${ }^{29}$. Organic photodetectors (OPDs) are very attractive for this application as they are inexpensive and can be flexible showing a promising alternative to silicon-based photodetectors ${ }^{25}, 30$. Organic photodetectors show a strong absorption coefficient, enabling the operation with thin layers. Furthermore, these materials can be tuned to improve the absorption spectra selectivity, as well as the optical bandgap and the electronic transport properties ${ }^{31-32}$. Most importantly, organic materials are advantageous in terms of processing since they can be formulated as inks and fabricated by printing technologies, which reduces photodetectors $\operatorname{cost}^{13,32}$.

There are two different approaches for medical X-ray detection systems classified as direct and indirect methods ${ }^{33}$. The direct method uses photoconductive materials with high $\mathrm{X}$ ray sensitivity and typically requires high operation bias voltage. The indirect method uses scintillator materials that absorb the X-ray radiation and convert it into visible light ${ }^{34}$. The 
photodetectors sensitive to visible light convert the light from the scintillator located on top of them into electric signals, thus allowing the indirect detection of X-ray radiation.

Both direct and indirect methods still face limitations and high costs when large areas or flexible applications are required. Polymer composites based on scintillator nanoparticles allow the design and overcome such limitations as well as innovative, novel applications thanks to their high efficiency, flexibility and low-cost production ${ }^{35}$.

Several works have explored the use of photodetectors for indirect X-ray detection based either on polymer thin-films filled with scintillator and fluorescence molecules ${ }^{35-36}$, inorganic high- $\mathrm{Z}$ nanocomposites ${ }^{37}$ or carbon nanotubes ${ }^{38}$ to enhance the sensitivity to $\mathrm{X}$ rays and consequently reduce the radiation dose. However, consistent approaches are still required to reach unfailing and reproducible deposition of multilayer stacks of diff erent functional materials.

In this work, a novel device for X-ray indirect detection was developed based on a thinfilm transistor organic photodetector architecture fabricated by inkjet printing and screen printed scintillator inks. A further interesting feature for applications is that the devices were manufactured in a standard laboratory environment (no cleanroom) and at low temperatures compatible with polymer substrates.

\section{Experimental details}

\subsection{Materials for the fabrication of the photodetectors}

Polyethylene naphthalate (PEN) films (Dupont Teijin Q65FA) with a thickness of $125 \mu \mathrm{m}$ were employed as flexible polymeric substrates. UTDAgIJ1 conductive silver nanoparticle ink from UT DOTS (UT Dots Inc., USA) was applied for the manufacturing of the electrodes. The applied dielectric material was cross-linked poly-4-vinylphenol (c-PVP, PVP purchased from Sigma Aldrich with a Mw. of about 25000). PVP was dissolved at room temperature in $10 \mathrm{~mL}$ propylene glycol monomethyl ether acetate (PGMEA), and 
magnetically stirred for 3 hours. Poly(melamine-coformaldehyde) methylated (PMFM, from Sigma Aldrich, Mw. of about 432, 84 wt.\% in 1-butanol) was added as a crosslinking agent under stirring for $2 \mathrm{~h}$. The ratio of PVP to PMFM was 2:1. Before printing, the conductive silver nanoparticle and the dielectric inks were filtered through $0.2 \mu \mathrm{m}$ syringe filters to ensure the removal of residual agglomerations. FS0096, a p-type polymer from Flexink (Flexink Ltd., UK) dissolved in mesitylene and tetralin mixture was used as amorphous organic semiconductor (OSC).

\subsection{Fabrication of the printed photodetectors.}

Inkjet printing was performed with a Dimatix Materials Printer (DMP) 2831 (Fujifilm

Dimatix Inc., USA), equipped with a 16 nozzles piezoelectric printhead with a nominal drop volume of about $10 \mathrm{pL}$ and a nozzle-to-nozzle spacing of $254 \mu \mathrm{m}$. For all the inks, the printing process was carried out with a maximum jetting frequency of $5 \mathrm{kHz}$ and a welladjusted, optimized control signal (waveform) for each individual ink formulation, as represented in figure 1. 


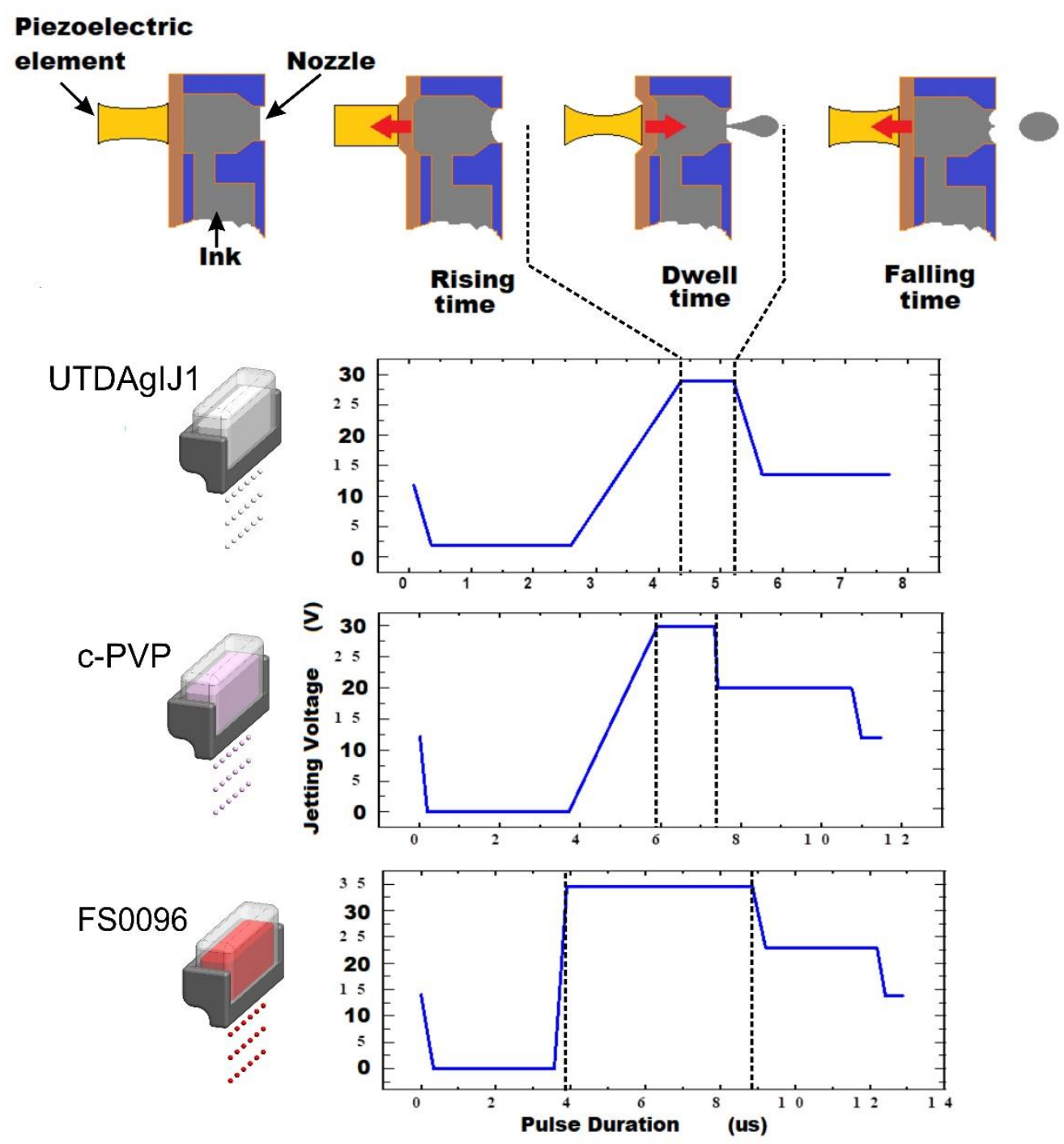

Figure 1. Silver nanoparticles, dielectric and OSC ink jetting waveform.

Figure 1 shows the optimized jetting waveforms used for the different ink formulations. The adjustment of the print waveform can be divided into three parts, represented in Figure 1: rising time, dwelling time and falling time. The rising time setting allows to define the amount of ink that will be ejected through the nozzle, defining the drop size. The setting of the dwelling time parameters allows to define how the drop leaves the nozzle. Finally, it is necessary to define the shape of the falling time, corresponding to the process of separating the drop from the nozzle, this step being critical to ensure that there is no formation of 
satellite drops. The maximum drop ejection voltage was $35 \mathrm{~V}$ and the drop ejection frequency was limited to $5 \mathrm{kHz}$ for higher repeatability.

Figure 2a shows the organic thin-film transistor (OTFT) architecture of the printed photodetectors consisting of four different layers: (i) a bottom gate layer using the silver nanoparticle ink, (ii) an insulating dielectric layer based on c-PVP, (iii) 12 interdigitated source-drain (S-D) electrodes using the silver nanoparticle ink, and (iv) a semiconducting layer on top of the stack. Further, the 5 x 5 sensors matrix layout is presented in Figure $2 \mathrm{~b}$. The spacing between S-D electrode fingers in the digital design was $120 \mu \mathrm{m}$ and represents the channel length of the TFT. The width of the individual finger electrodes in the design was $26000 \mu \mathrm{m}$ and the channel length was $40 \mu \mathrm{m}$.

a)

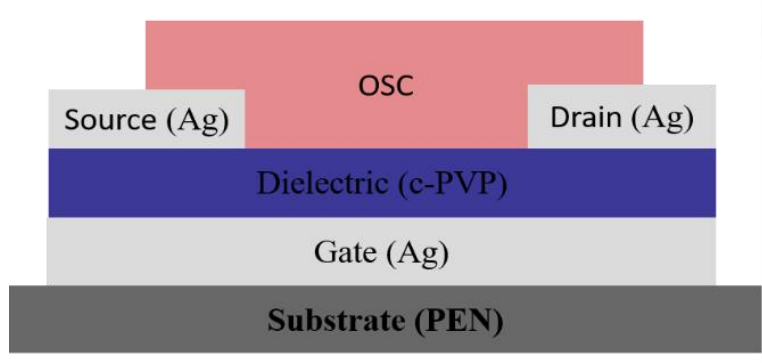

\section{b)}

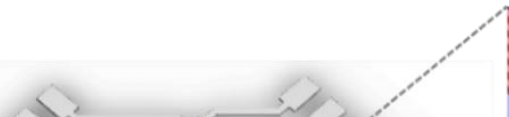

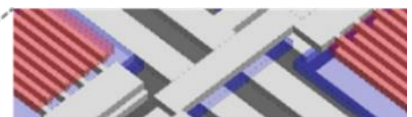

Figure 2. a) Schematic image of the TFT-based photodetector device structure; b) layout of the inkjet printed $5 \times 5$ photodetector matrix.

Before printing, the PEN substrate was cleaned with ethanol and then dried by a nitrogen flow to remove any remaining particles. For the deposition of the bottom gate, the silver ink was jetted using a print resolution of $635 \mathrm{dpi}$ that corresponds to a drop space of $40 \mu \mathrm{m}$. During the process of printing, the cartridge and substrate temperature was set to $35^{\circ} \mathrm{C}$ and $40{ }^{\circ} \mathrm{C}$ respectively. The post-printing procedure for the printed silver layer consisted of 
drying and sintering in an oven (JP Selecta 2005164) at $150{ }^{\circ} \mathrm{C}$ for 30 minutes. Subsequently, two layers of c-PVP were printed using a print resolution of 1270 dpi (drop space of $20 \mu \mathrm{m}$ ), with both the substrate and printhead temperature set to room temperature $\left(\sim 28^{\circ} \mathrm{C}\right)$. Finally, curing of the dielectric layer took place at $150{ }^{\circ} \mathrm{C}$ for 30 minutes in an oven. For the S-D electrodes, the silver nanoparticle ink was jetted with print resolution of 635 dpi and just 1 nozzle, in order to achieve higher print accuracy. The post-printing treatment of the silver layer was the same as indicated earlier. The final layer for the OTFT stack (photodetector) was the OSC, that was printed with a substrate temperature of $35^{\circ} \mathrm{C}$ and printhead temperature set to room temperature $\left(\sim 28{ }^{\circ} \mathrm{C}\right)$. Then, the layer was cured in an oven at $100{ }^{\circ} \mathrm{C}$ for 10 minutes. During the printing, the used drop space was $20 \mu \mathrm{m}$ and the quality check of the printed patterns was performed using the microscope Leica DM4000 M.

\subsection{Development and application of the scintillator ink}

A polymer based scintillator ink formulation based on gadolinium oxide doped with europium $\left(\mathrm{Gd}_{2} \mathrm{O}_{3}: \mathrm{Eu}^{3+}\right)$ as scintillator material and the fluorescence molecules 2,5dipheniloxazol (PPO) and 1,4-bis-(2-(5-phenioxazolil))-benzol (POPOP) was formulated following the procedure described in ${ }^{36} . \mathrm{Gd}_{2} \mathrm{O}_{3}: \mathrm{Eu}^{3+}$ was selected as scintillator nanoparticles due to its, high density $\left(\approx 7.4 \mathrm{~g} \cdot \mathrm{cm}^{-3}\right)$, high atomic number and good light

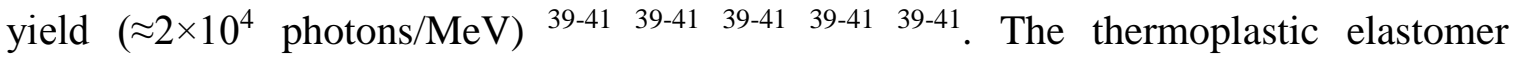
copolymer Styrene-Ethylene/Butylene-Styrene (SEBS) Calprene CH-6120, with a molecular weight of $245.33 \mathrm{~g} / \mathrm{mol}$ and a ratio of Ethylene-Butylene/Styrene of 68/32, supplied by Dynasol was used as the polymer matrix to develop a flexible ink with suitable adhesive properties. It has been proven that this combination of materials is able to efficiently convert $\mathrm{X}$-ray radiation into visible light ${ }^{34}$. Thus, the formulation of the 
scintillator ink was as follows: 0.5 wt.\% of $\mathrm{Gd}_{2} \mathrm{O}_{3}: \mathrm{Eu}^{3+}$ scintillator nanoparticles (Nanograde) and 1 wt\% of PPO (Sigma-Aldrich D210404) and 0.01wt.\% of POPOP (Sigma-Aldrich P3754) were added to toluene (Panreac with a density of $0.86 \mathrm{~g} / \mathrm{cm}^{3}$ at 20 ${ }^{\circ} \mathrm{C}$ ) and placed for dispersion in an ultrasound bath for 3 hours. After the complete dispersion of the scintillator nanoparticles and fluorescence molecules, SEBS was added with a ratio of $1: 4$ ( $1 \mathrm{~g}$ of polymer was added to $4 \mathrm{ml}$ of solvent) to the solution and placed in a Teflon ${ }^{\mathrm{TM}}$ mechanical stirrer at $150 \mathrm{rpm}$ until complete dissolution and homogeneous mixing of all components. Finally, a scintillator ink formulation was obtained with a viscosity between 2 and 5 Pa.s suitable for screen printing.

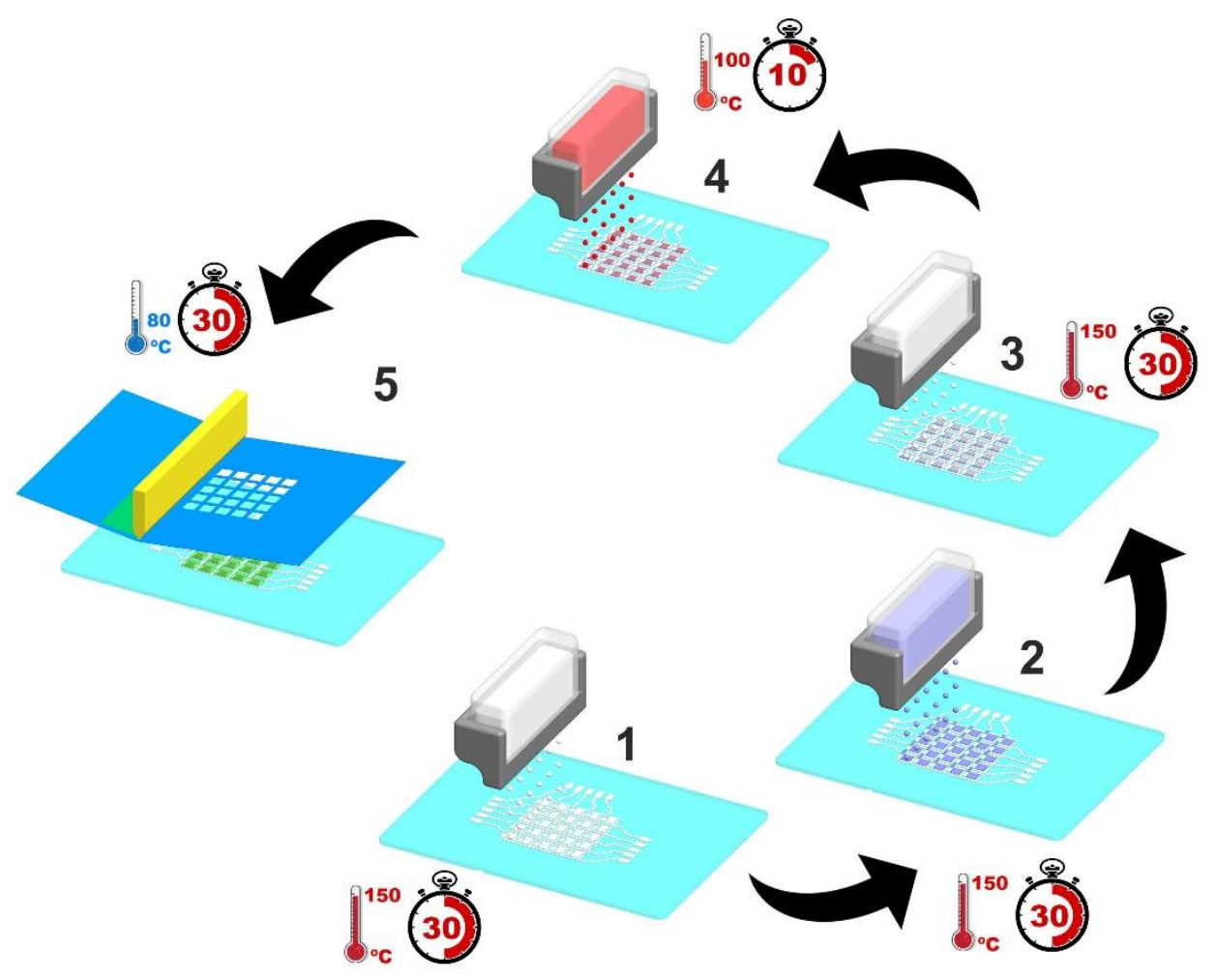

Figure 3. Schematic representation of the printing processes used for the fabrication of the photodetector array and respective cure conditions: inkjet printing of 1) silver bottom layer; 2) dielectric layer of c-PVP ink; 3) silver layer corresponding to the drain and source electrodes; 4) OSC layer and screen-printing of 5) the scintillator ink. 
The scintillator ink was deposited on top of the photodetectors by the screen printing technique as represented in Figure 3 - process 5, using a home-made manual screenprinting machine. The screen-printer was fabricated in stainless steel and presents a support for the substrate that can be adjusted on the $\mathrm{x}$ and $\mathrm{y}$ axis. Further, it shows a frame to add and fix the screen mesh that can be adjusted in the $\mathrm{z}$ axis to ensure the required distance to the substrate support. A polyester mesh screen was used in the printer. The dimensions of the aluminum frame attached to the mesh was $450 \times 350 \mathrm{~mm}^{2}$ with a profile of $20 \times 20 \mathrm{~mm}^{2}$. The mesh count for the printing screen was 65 threads $/ \mathrm{cm}$, the thread diameter was $52 \mu \mathrm{m}$, and the square-edged mesh opening was $102 \mu \mathrm{m}$. The squeegee orientation angle was set to $45^{\circ}$ relative to the print substrate and the tension on the mesh was $20 \mathrm{~N}$. After printing, the samples were oven-cured at $80{ }^{\circ} \mathrm{C}$ for 30 minutes. Figure 3 summarizes the different steps for the fabrication of the X-ray detection devices.

\subsection{Characterization of the printed photodetectors}

Scanning electron microscopy (SEM) images were obtained with a Zeiss Auriga microscope. The system is equipped with a focused ion beam (FIB) tool Zeiss 1560XB Cross Beam. FIB cuts were performed to obtain cross-sectional images of the layer stack in order to determine the thickness and the structure of the individual layers. Micro Raman spectroscopy experiments were performed under green and infrared laser excitations, 514.7 $\mathrm{nm}$ and $532 \mathrm{~nm}$, and $785 \mathrm{~nm}$, respectively. Absorption spectra of the active layers were obtained by using the UV/Vis microreflectance spectrometer TIDAS S MSP 800 (J\&M Analytik AG, Esslingen, Germany) connected to a Zeiss Axio Imager M2m microsope. The light source of the microscope was used to illuminate the samples through the microscope objective lenses. The reflected light was collected by the same lenses and guided to the spectrometer by optical fibres. The electrical characterization of the 
photodetectors is equal to the electrical characterization of an organic thin-film transistor (OTFT). In order to ensure the uniformity and reproducibility of the results, the functionality of the printed photodetectors array was analysed for all 25 OTFT within the array. The percentage of functional OTFTs was over $80 \%$ relative to the total number of printed OTFT. This is also the same for different printed OTFT arrays. Furthermore, the photodetector and especially the active, semiconducting layer was exposed to a light source as shown in Figure 4.

a)

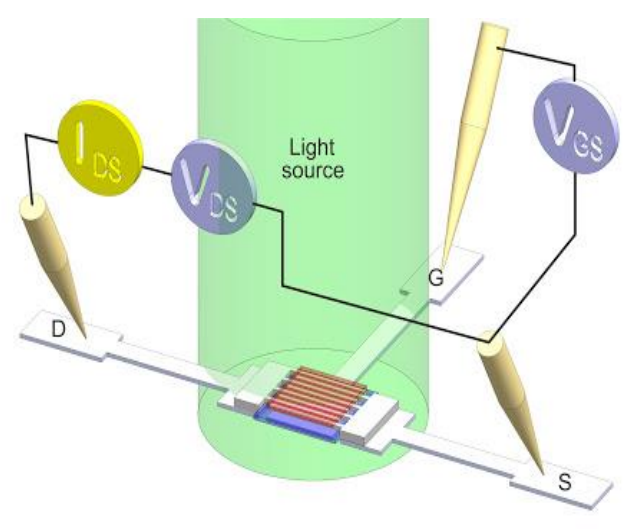

b)

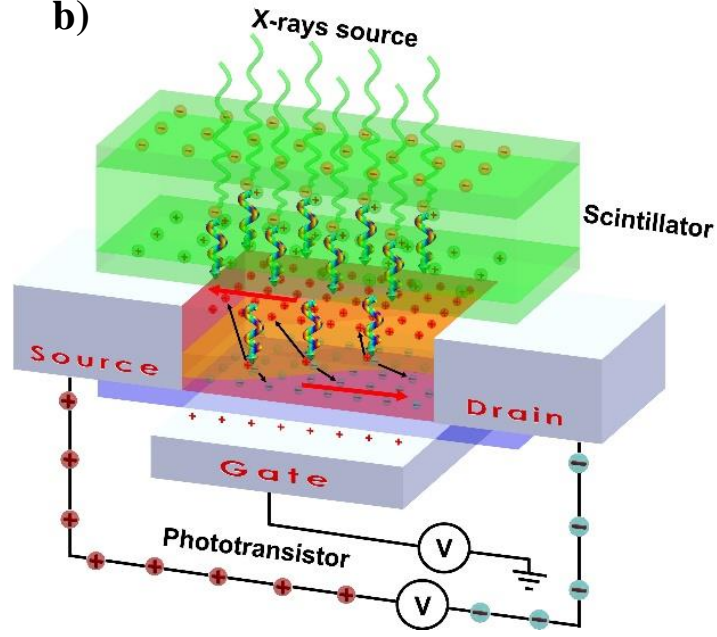

Figure 4. a) Schematic representation of the electrical and functional characterization setup of the transistor-based photodetectors for X-ray detection. The OTFT characterization parameters, voltage drain-source $\left(V_{D S}\right)$, current drain-source $\left(I_{D S}\right)$ and voltage gate-source $\left(\mathrm{V}_{\mathrm{GS}}\right)$ were measured, using the terminals drain $(\mathrm{D})$, source $(\mathrm{S})$ and gate $(G)$; b) schematic representation of the experimental procedure with the final device, with the scintillator placed on top of the photodetectors, and representation of the main physical process taking place in the all-printed device. 
The electrical measurements were performed with the QuadTech 1920 Precision LCR Meter. The electrical operation properties of the printed photodetectors were characterized with a 2400 source/meter and a 6457 picoammeter with integrated voltage source from Keithley (Keithley Instruments, Cleveland, OH, USA) controlled by LABView program developed for current vs. voltage (I-V) characteristic measurements. The probe station was directly connected to the photodetector using gold-coated round tips. The IEEE1620 standard test method was used for the characterization of the organic transistors. All measurements were performed at room temperature, in dark and multi-wavelength light conditions, achieved with a broadband Xenon arc lamp (Xenon XBO 75W/2 OFR, Osram, Munich, Germany) coupled via a 44-position bandpass filter wheel and appropriate focusing optics to a $100 \mu \mathrm{m}$ core diameter multimode fibre, that allows to obtain a wavelength variation between $362 \mathrm{~nm}$ and $1000 \mathrm{~nm}$. The $\mathrm{I}_{\mathrm{DS}}-\mathrm{V}_{\mathrm{DS}}$ curve was obtained applying a fixed $\mathrm{V}_{\mathrm{GS}}$ when a light source was focused on the active area of the photodetector. The process was made for each wavelength (between $400 \mathrm{~nm}$ to $700 \mathrm{~nm}$ ) and for each different applied $\mathrm{V}_{\mathrm{GS}}$ (from 5 to $45 \mathrm{~V}$ ).

The performance of the printed photodetector under X-ray radiation with and without the printed scintillator layer was measured by subjecting the samples to the X-ray radiation produced by a Bruker D8 Discover diffractometer using $\mathrm{Cu} \mathrm{K} \alpha$ incident radiation (wavelength of 1.54056 angstroms) and powered with a voltage of $40 \mathrm{kV}$ and a current ranging from 0 to $40 \mathrm{~mA}$ (output power changes from 0 to $1600 \mathrm{~W}$ ). The efficiency of the X-ray to visible conversion was evaluated with an electronic measurement system that allows to quantify the emitted visible wavelength radiation, as described in ${ }^{35}$.

\section{Results and discussion}


The microstructure and the thickness of the printed layers for the printed photodetectors were obtained by the scanning electron microscopy (SEM) as shown in Figure 5 a). The cross-sectional images were obtained by partial material removal using a focused gallium ion beam (FIB).
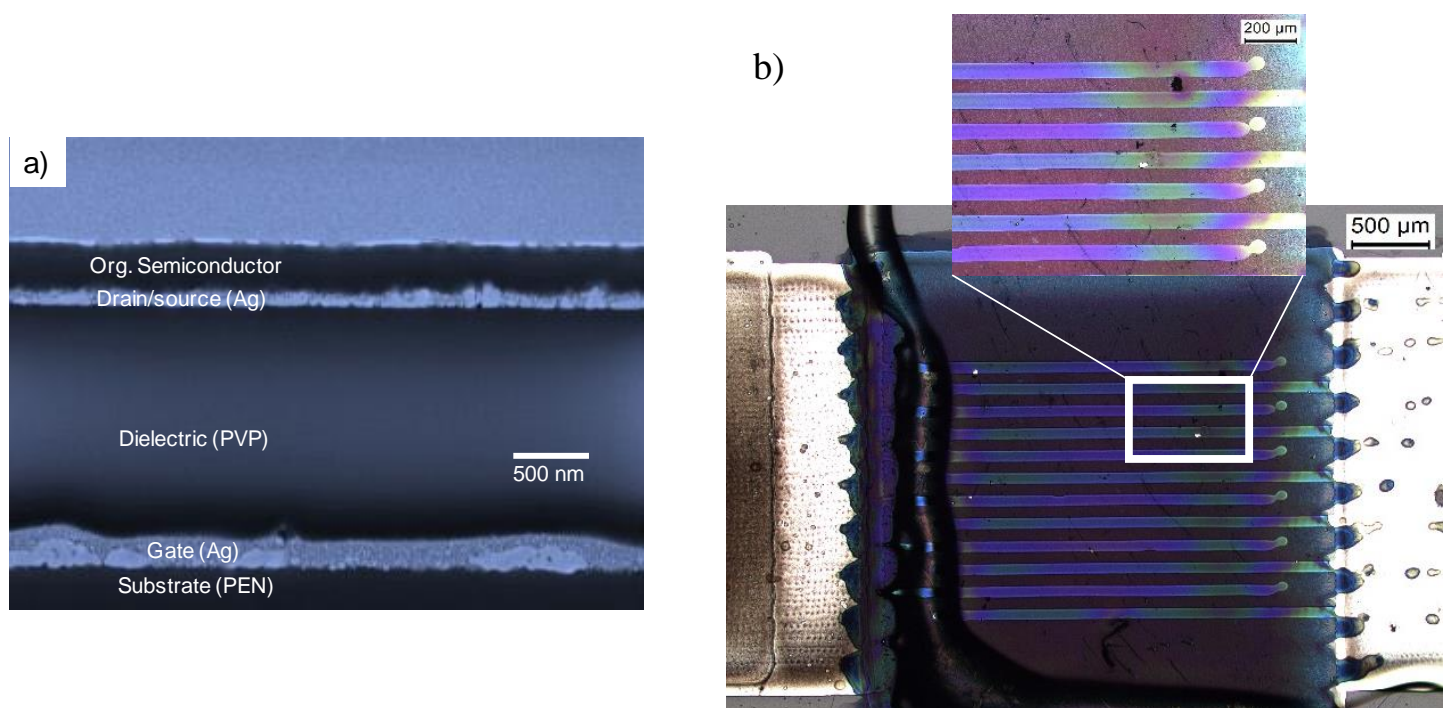

Figure 5. a) Cross-section analysis by scanning electron microscopy of the organic printed photodetector showing the different layers of the heterostructure. b) photograph of the TFT channel with a width of about $26000 \mu \mathrm{m}$ and a channel length of about 57 $\mu \mathrm{m}$.

In Figure 5 a), it is observed that the silver electrodes are comparably rough and depending on the lateral position with different microstructural morphology, e.g. silver nanoparticle agglomerations are observed along the layer. The average layer thickness of the gate electrode is about $200 \pm 40 \mathrm{~nm}$. The average layer thickness of the S-D electrodes is just half of the gate electrode with about $100 \pm 30 \mathrm{~nm}$. The dielectric has a thickness of about $1500 \pm 30 \mathrm{~nm}$ and the organic semiconductor a thickness of about $300 \pm 30 \mathrm{~nm}$. The different layer thicknesses are caused by the substrate ink interaction of the different ink formulations and by the different print parameters indicated in the experimental section. 
The UV/Vis micro-reflectance spectroscopy was performed for the OSC layer and is show in figure 6a. The OSC layer absorbs radiation with a wide and large band in the range of approximately $550 \mathrm{~nm}$ to $700 \mathrm{~nm}$ and narrower less intense bands below $430 \mathrm{~nm}$ and in the range of about 830 to $950 \mathrm{~nm}$.

The interface between the metal contact electrode, the dielectric and the organic semiconductor at the device channel plays a crucial role with regard to the performance of any organic semiconductor device ${ }^{42}$. In organic photodetectors, a field in which electrons and holes play various roles in determining the functionality and performance of organic devices, e.g., charge injection/extraction, charge separation, and charge transport are highly dependent on the quality and reliability of the interface of the printed devices. Carrier injection from metal electrodes to semiconductors and its electrical transport are important key factors in electronic devices ${ }^{43-45}$. Thus, the choice of the electrodes is as important as the selection of organic semiconductor (OSC). In order to evaluate the metal electrode/polymer interface, Raman spectroscopy was performed (Figure 6b). It was observed that the interaction with the metal electrode affects some of the Raman modes marked by asterisks $(*)$ in Figure 6b. It is also observed from the photoluminescence (PL) spectra averaged over the channel and electrode regions (excitation wavelength of PL spectra is $532 \mathrm{~nm}$ ), Figure $6 \mathrm{c}$ and Figure $6 \mathrm{~d}$, respectively, that the photon emission from the organic semiconductor gets quenched for the polymer located at the electrode region. This slight quenching is spectrally selective affecting the emission band located around $733 \mathrm{~nm}$. This is contrary to the case of the band at $775 \mathrm{~nm}$ that experiences a slight increase over the electrode region with respect to the channel. Figure 6e shows the PL intensity map averaged over the emission range from $660 \mathrm{~nm}$ to $830 \mathrm{~nm}$, where the PL quenching over the electrode at the bottom side of the image is clearly visible. In figure $6 f$ it is shown the intensity ratio map between the bands at $733 \mathrm{~nm}$ and $778 \mathrm{~nm}$. These results are particularly 
interesting because they show a way to increase the light emission and so the performance of the device as an X-ray detector, for example, by decreasing the lateral dimensions of the interdigitated electrodes. Indeed, the Ag electrodes act as a pool of charge carriers that result in a de-doping effect on the polymer quenching the emission band ${ }^{46}$. According to the position of Raman modes that are affected, we can tentatively attribute these vibrations
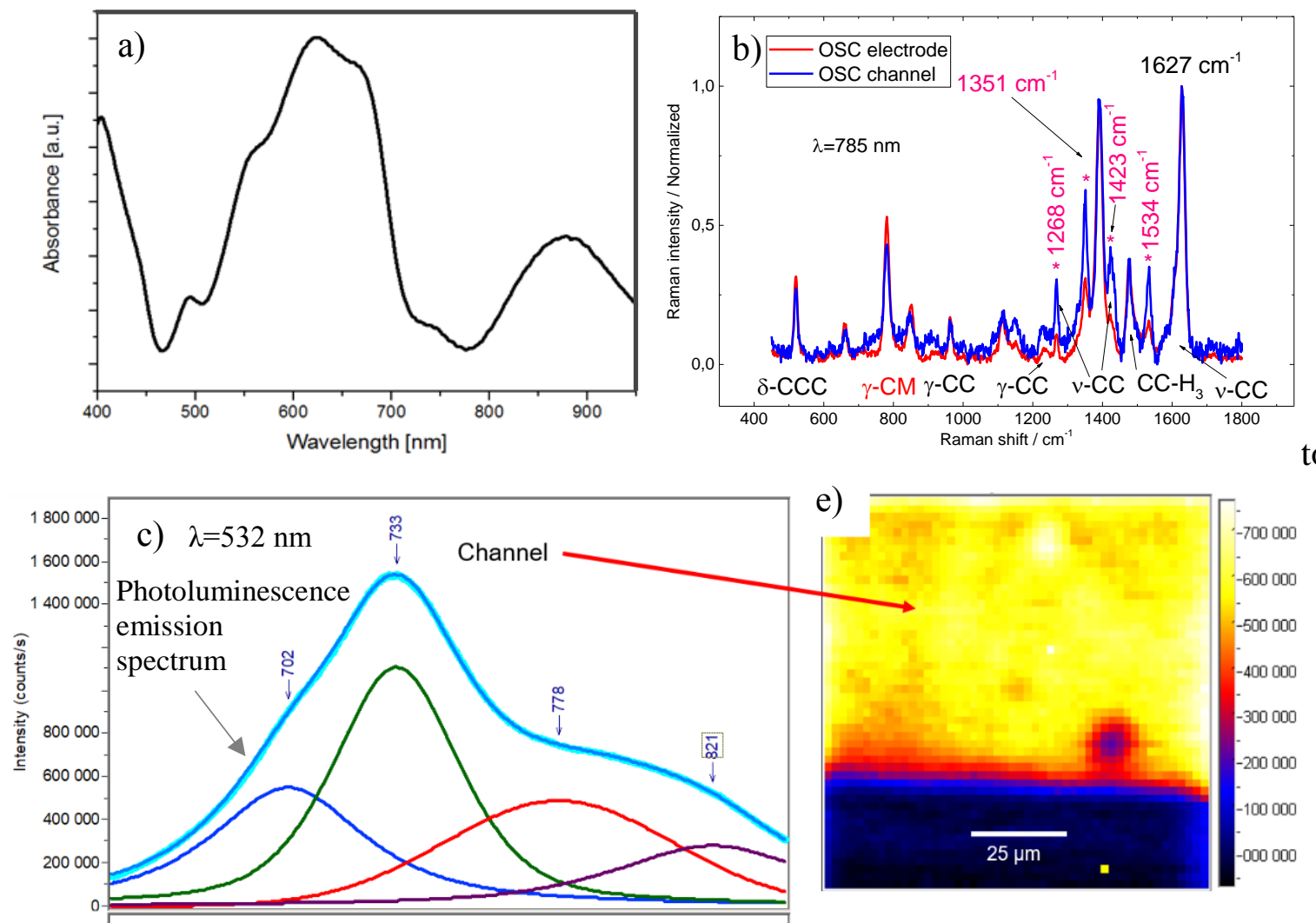

e)

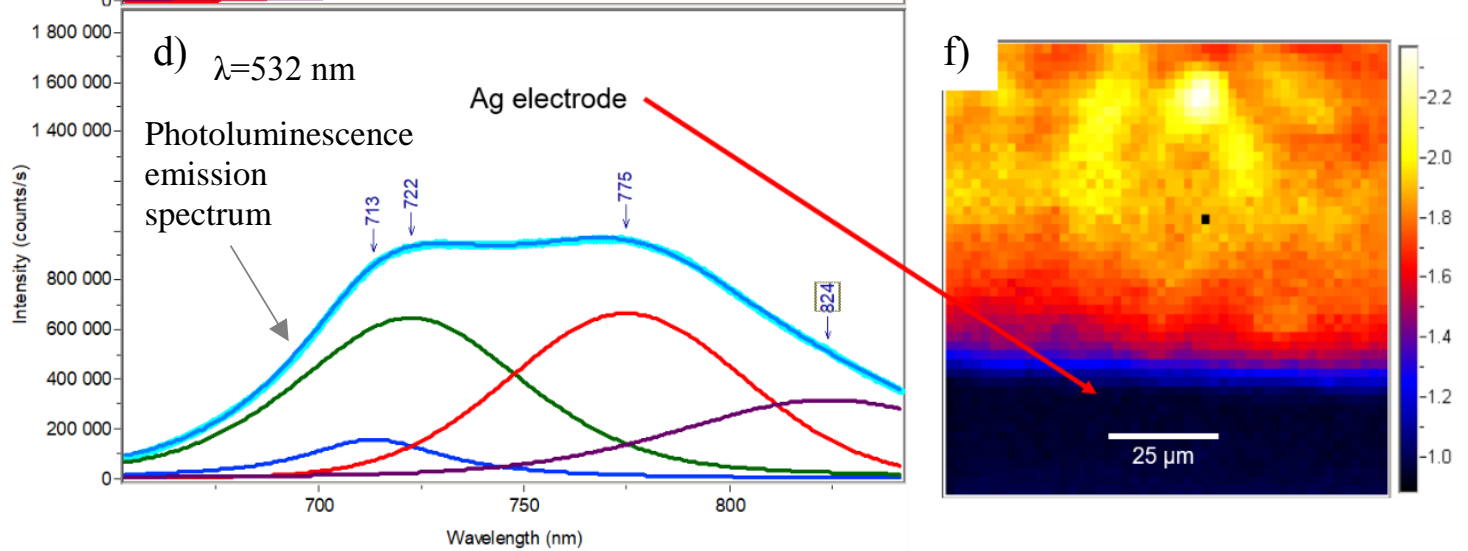

the carbon vibrations shown in figure $6 \mathrm{~b}$.

Figure 6. a) UV/Vis micro-reflectance spectra of OSC layer; b) Raman spectra averaged over the Ag electrode and channel regions. The different modes affected by the interaction 
of the polymer with the electrode are marked by asterisks $(*)$; The photoluminescence emission spectra (light blue curve) averaged over the channel region c) and the electrode region d) with different modes deconvolved by fitting with 4 different emission bands (blue, red, green and purple curves); e) The emission map shown for the intensity within the spectral region around $733 \mathrm{~nm}$. f) Map obtained from the intensity ratio between the emission around $733 \mathrm{~nm}$ and $775 \mathrm{~nm}$.

The absorbance spectrum of the OSC layer is very important for the operation principle of photodetector, since the indirect detection method is used with a scintillator, positioned or printed over the photodetector array, converting X-ray radiation in visible light ${ }^{47}$. The emitted visible light from scintillator interacts with the active material of the photodetector and should be converted into electron-hole pairs, which represents the imaging charge ${ }^{48}$. Therefore, the OSC layer of the printed photodetector needs to generate photo-activated charge carriers because of the radiation of the scintillator. Most of the available OSC with Highest Occupied Molecular Orbital - Lowest Unoccupied Molecular Orbital (HOMOLUMO) band gap energy ranging from $1.5-3.0 \mathrm{eV}$ show photo-induced charge carrier effects in the visible light spectrum of about $400 \mathrm{~nm}$ to $700 \mathrm{~nm}$. Based on the maximum absorbance of the OSC layer, the band gap energy is about $1.70 \mathrm{eV}$.

The photodetector needs to be functional in the range of the visible radiation wavelength. Thus, a correct selection of the OSC is necessary which should be suitable for the required application and the detected light should also have a matching within a wavelength range that matches the absorption spectrum of the active layer and scintillator material ${ }^{49-50}$. In this case, the OSC should be suitable to wavelengths on the visible light range. This fact is due to the absorption/emission spectra and quantum efficiency of the scintillator material, which converts the X-ray radiation into visible radiation. During the scintillation process, 
several relaxation processes occurs and leads to a large number of relaxed electron-hole pairs which are transferred to states of lower energy and the corresponding emission of light (Figure 4b). Then, the electrons migrate to the activator excited state and the holes in the valence band migrate to the activator ground state ${ }^{51}$. These transitions leads to the production of a scintillation photon ${ }^{51}$. The fluorescence molecules (POPOP and PPO) absorb this scintillation energy and re-emits at higher wavelength, in the visible wavelength range that should be detected for the active material of the photodetector, inducing the photogenerated charge carriers in the OSC. Under a potential difference (external bias on the device) those carriers will result in a current increase, as represented on Figure $4 \mathrm{~b}$. To evaluate the electrical response and performance of the printed photodetectors, the I-V characteristics were measured in dark and under illumination. The wavelength for the illuminated measurement method (about $630 \mathrm{~nm}$ ) was selected in order to match the maximum absorbance of the active layer present in the photodetector, in order to absorb in the spectral region were the emission of the scintillating material. The spectral peak of the photodetector absorbance should be matching with the visible light radiation as an assumed requirement to maximize application sensitivity to the application ${ }^{1,52}$. 

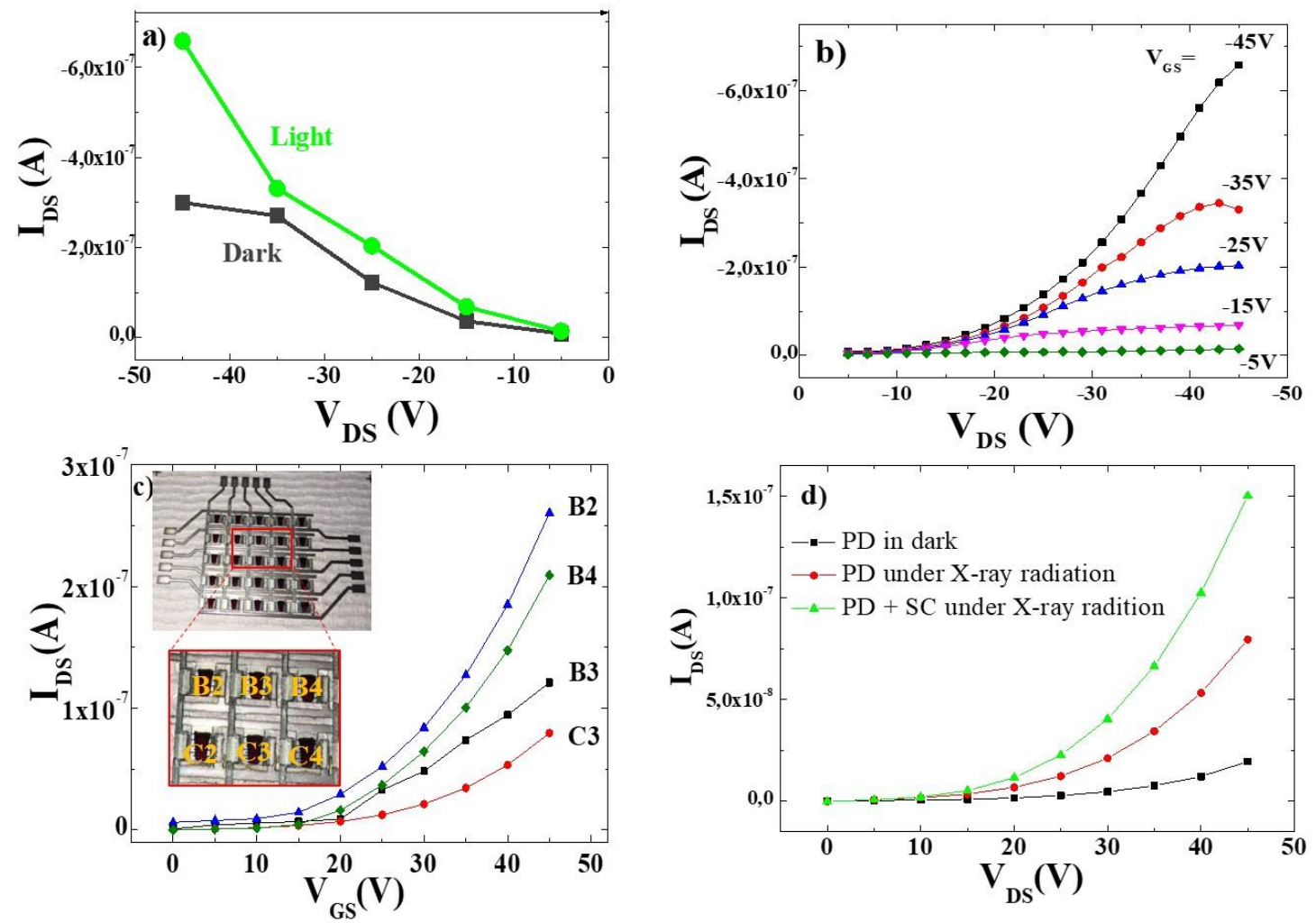

Figure 7. a) I-V characteristics of the printed photodetectors (PD) measured in dark and under light radiation $(630 \mathrm{~nm})$ as a function of the applied drain-source voltage; b) I-V output curved for the PD under illumination; c) Electrical response of randomly-selected PD from the 5 x 5 TFT matrix (channel: length of $57 \mu \mathrm{m}$, width of $26000 \mu \mathrm{m}$ (image in the inset)), under X-ray radiation $\left(\mathrm{P}_{\mathrm{X} \text {-ray beam }}=1600 \mathrm{~W}\right.$ and a voltage applied on system of $\left.45 \mathrm{~V}\right)$, as a function of the gate-source voltage; d) I-V curves of one of the single PD present in the PD array in dark (black curve), and under X-ray radiation with (green curve) and without (red curve) scintillator (SC) film (B2, B3, B4 and C3 are the nomenclature used to identify each PD: the different PD on the array are identified by a letter, which corresponds to the line, and with a number, which indicates the column of the $5 \times 5$ PD array), as a function of the drain-source voltage $\left(\mathrm{P}_{\mathrm{x} \text {-ray beam }}\right.$ $=1600 \mathrm{~W}$ and a voltage applied bias of $45 \mathrm{~V})$.

Figure 7a shows the electrical characteristics of the photodetector array obtained from I-V measurements in dark and under light radiation with a wavelength of about $630 \mathrm{~nm}$. At $45 \mathrm{~V}$ bias, the dark current was about $300 \mathrm{nA}$, while under illumination the photocurrent 
was more than doubled with about $660 \mathrm{nA}$. It is concluded that the photodetector under illumination shows an increase in the drain-source current of approximately $120 \%$ with respect to the device under dark. This indicates a good performance of the printed device to be used as a photodetector, relative with the photodetectors present in literature, which present photocurrents in the range of 20 to $200 \mathrm{nA}^{23,53-54}$. The wavelength of illumination is in the spectral range response of the active layer (OSC layer) present on the photodetector array. The performance of the photodetector array was measured considering different applied negative voltages, $5,15,25,35$ and $45 \mathrm{~V}$, respectively. Figure $7 \mathrm{~b}$ shows that the printed photodetector array has its maximum response at $45 \mathrm{~V}$.

After that, the electrical response of the photodetector array (PD in Figure 7. Image in the inset of Figure 7c) was evaluated under X-ray radiation. The Figure 7c shows that the photodetector matrix has a consistent response, with the same electrical behavior when the radiation was applied independently of the single pixel, i.e. when the radiation is applied to every single photodetector, the electrical response is the same, independently of the photodetector within the array. The consistent response demonstrates the uniformity of the photodetectors response within the array, which is also extended to different photodetector arrays, as expected due to the reproducibility of printing technologies for device fabrication. For the indirect detection, the scintillator material used (SC in the graph, Figure 7d) was printed on top of the photodetector matrix by screen printing technology as described in experimental section. The printed film has a thickness of around $30 \mu \mathrm{m}$ and a transmittance of about $70 \%$, measured by Ultraviolet-Visible (UV-VIS) spectroscopy in a range between 200 to $800 \mathrm{~nm}$ with a $1 \mathrm{~nm}$ step in a UV-2501PC spectrometer, in order to allow the light passing through it to the OSC layer. The intensity of the converted visible radiation of this scintillator printed film increases with increasing X-ray output power. The scintillator nanoparticles content was optimized for this specific scintillator based on the 
work already reported ${ }^{36}$ in order to have a higher number of produced visible photons ${ }^{55}$. It is to notice that other scintillator nanoparticles could be used, such as $\mathrm{Gd}_{2} \mathrm{O}_{2} \mathrm{~S}: \mathrm{Tb}^{56}$, $\mathrm{Lu}_{2} \mathrm{O}_{3}: \mathrm{Eu}^{57}$ and $\mathrm{Gd} 2 \mathrm{O} 2 \mathrm{~S}: \mathrm{Eu}^{58}$ but, in this case, the specific scintillator output spectra should be taken into consideration to design and fabricate the specific photodetector in order to maximize visible to electrical signal conversion. Figure $7 \mathrm{~d}$ shows the response of the photodetector under X-ray radiation. The conditions of the X-ray power, voltage of $40 \mathrm{kV}$ and a power from 0 to $1600 \mathrm{~W}$ were optimized based on previous reported works ${ }^{34-}$ ${ }^{35}$. In the following, the results with a $\mathrm{P}_{\mathrm{x} \text {-ray beam }}$ of $1600 \mathrm{~W}$ are shown. At lower $\mathrm{x}$-ray power, the trend is the same as at $1600 \mathrm{~W}$. After analysing the stability of the of the device upon ten X-ray radiation on/off cycles, the printed photodetector shows a stability of the signal of $98 \%$ for $\mathrm{V}_{\mathrm{DS}}$ from 20 to $45 \mathrm{~V}$, being therefore the variations within experimental error and therefore, no radiation aging is detected. The current intensity shows an increase with increasing applied voltage. At higher voltages (45 V) a higher electrical response of the photodetector under $\mathrm{X}$-ray radiation (53 $\mathrm{nA}$ ) is observed in comparison with the photodetector in dark (19 nA). This increase is more pronounced when the scintillator material is on top of the photodetector $(102 \mathrm{nA})$. This represents an increase of the current intensity of about $93 \%$ and proves the good performance of the printed scintillator material. The printed X-ray detector responsivity is approximately $1.457 \mu \mathrm{A} / \mathrm{W}$, under X-ray irradiation. Thus, in this work the development of a novel generation of flexible, all printed, and large-area devices applied on fields like security or medical imaging, for the detection of X-ray radiation was successfully demonstrated.

\section{Conclusions}


The development of a novel generation of all printed and flexible indirect X-ray detectors based on polymer based scintillator inks and inkjet printed photodetector TFT-based array has been successfully demonstrated.

The electrical response of the photodetector shows a dark current about $300 \mathrm{nA}$, while under illumination the photocurrent was about $660 \mathrm{nA}$, which corresponds to an increase of around $120 \%$, demonstrating the good performance of the printed device as a photodetector. Regarding to the performance as X-ray detection, after printing the scintillator ink, it was observed a higher electrical response of the photodetector under Xray radiation $(53.1 \mathrm{nA})$ in comparison with the photodetector in dark (19.5 nA). This increase is strongly improved when the scintillator material is on top of the photodetector (102.4 nA). This increase of $93 \%$ in the current intensity of the device under X-ray radiation shows the suitability of the printed scintillator and all the devices for applications in the X-ray radiation detection area, concerning the results already reported ${ }^{29,59}$ of different photodetectors for indirect X-ray detection. Thus, a novel all-printed X-ray detection device for indirect radiation detector was manufactured with a combination of two different printing methods, inkjet and screen printing. These devices show a very promising performance for emerging large-area and low-cost flexible optoelectronics, which can be applied to, e.g., medical imaging or security industry.

\section{AUTHOR INFORMATION}

\section{Corresponding Author}

*joliveira@fisica.uminho.pt, and senentxu.lanceros@bcmaterials.net

\section{Author Contributions}


The manuscript was written through contributions of all authors. All authors have given approval to the final version of the manuscript.

\section{Funding Sources}

FCT- Fundação para a Ciência e Tecnologia

Basque Government Industry

\section{ACKNOWLEDGMENT}

The authors thank FEDER funds through the COMPETE 2020 Programme and National Funds through FCT - Portuguese Foundation for Science and Technology under Strategic Funding UID/FIS/04650/2013 and projects PTDC/EEI-SII/5582/2014, PTDC/CTMENE/5387/2014 and in the framework of EuroNanoMed 2016 call, Project LungChek ENMed/0049/2016. J.O. and V. C. thank the FCT for the SFRH/BD/98219/2013 and SFRH/BPD/97739/2013 grants, respectively. The authors acknowledge funding by the Spanish Ministry of Economy and Competitiveness (MINECO) through the project MAT2016-76039-C4-3-R. Financial support from the Basque Government Industry Department under the ELKARTEK program is also acknowledged. We thank Iain McCulloch and Martin Heeney from Flexink for providing the organic semiconductor. Dirk Rittrich (Department Layer Deposition at Fraunhofer ENAS) is acknowledged for the FIB/SEM analysis and the sample preparation. RDR acknowledges the DFG Unit FOR1317 SMINT, the Cluster of Excellence, and the Tomsk Polytechnic University Competitiveness Enhancement Program grant TPU CEP_IHTP_73|2017. This work was performed in the context of the European COST Action MP1302 Nanospectroscopy.

\section{ABBREVIATIONS}


TFT, thin-film transistor; DVD, digital versatile discs; CCD, charge-coupled device;

CMOS, complementary metal-oxide-semiconductor; OPDs, organic photodetectors; PEN,

Polyethylene naphthalate; PGMEA, propylene glycol monomethyl ether acetate; PMFM,

Poly(melamine-coformaldehyde) methylated; c-PVP, cross-linked poly-4-vinylphenol;

DMP, Dimatix Materials Printer; OSC, organic semiconductor; PPO, 2,5-dipheniloxazol;

POPOP, 1,4-bis-(2-(5-phenioxazolil))-benzol; SEBS, Styrene-Ethylene/Butylene-Styrene

(SEBS); OTFT, organic thin-film transistor; D, drain; G, gate; $S$, source; $\mathrm{V}_{\mathrm{DS}}$, voltage

drain-source; IDS, current drain-source; $V_{G S}$, voltage gate-source; FIB, focused ion beam;

SEM, scanning electron microscopy; PL, photoluminescence; PD, photodetector; HOMO-

LUMO, Highest Occupied Molecular Orbital - Lowest Unoccupied Molecular Orbital;

\section{References}

1. Yotter, R. A.; Wilson, D. M., A review of photodetectors for sensing light-emitting reporters in biological systems. IEEE Sensors Journal 2003, 3 (3), 288-303.

2. Spahn, M., X-ray detectors in medical imaging. Nuclear Instruments and Methods in Physics Research, Section A: Accelerators, Spectrometers, Detectors and Associated Equipment 2013, 731, 57-63.

3. Pacella, D., Energy-resolved X-ray detectors: The future of diagnostic imaging. Reports in Medical Imaging 2015, 8, 1-13.

4. $\quad$ Spahn, M., Flat detectors and their clinical applications. European Radiology 2005, 15 (9), 1934-1947.

5. $\quad$ Pani, S.; Saifuddin, S. C.; Ferreira, F. I. M.; Henthorn, N.; Seller, P.; Sellin, P. J.; Stratmann, P.; Veale, M. C.; Wilson, M. D.; Cernik, R. J., High energy resolution hyperspectral x-ray imaging for low-dose contrast-enhanced digital mammography. IEEE Transactions on Medical Imaging 2017, 36 (9), 1784-1795.

6. $\quad$ Cole, L. E.; Ross, R. D.; Tilley, J. M.; Vargo-Gogola, T.; Roeder, R. K., Gold nanoparticles as contrast agents in X-ray imaging and computed tomography. Nanomedicine 2015, 10 (2), 321-341.

7. Pais, A.; Banerjee, A.; Klotzkin, D.; Papautsky, I., High-sensitivity, disposable lab-on-a-chip with thin-film organic electronics for fluorescence detection. Lab on a Chip 2008, 8 (5), 794-800.

$8 . \quad$ De Fazio, D.; Goykhman, I.; Yoon, D.; Bruna, M.; Eiden, A.; Milana, S.; Sassi, U.; Barbone, M.; Dumcenco, D.; Marinov, K.; Kis, A.; Ferrari, A. C., High Responsivity, Large-Area Graphene/MoS2 Flexible Photodetectors. ACS Nano 2016, 10 (9), 8252-8262.

9. Xie, C.; Yan, F., Flexible Photodetectors Based on Novel Functional Materials. Small 2017, 13 (43).

10. Liu, N.; Tian, H.; Schwartz, G.; Tok, J. B. H.; Ren, T. L.; Bao, Z., Large-area, transparent, and flexible infrared photodetector fabricated using $\mathrm{P}-\mathrm{N}$ junctions formed by $\mathrm{N}$-doping chemical vapor deposition grown graphene. Nano Letters 2014, 14 (7), 3702-3708.

11. Wẹgrzecka, I.; Wẹgrzecki, M., Silicon photodetectors - The state of the art. Opto-electronics Review 1997, 1997 (2), 137-146.

12. Casalino, M.; Coppola, G.; De La Rue, R. M.; Logan, D. F., State-of-the-art all-silicon sub-bandgap photodetectors at telecom and datacom wavelengths. Laser and Photonics Reviews 2016, 10 (6), 895-921.

13. Pace, G.; Grimoldi, A.; Sampietro, M.; Natali, D.; Caironi, M., Printed photodetectors. Semiconductor Science and Technology 2015, 30 (10).

14. Baeg, K. J.; Binda, M.; Natali, D.; Caironi, M.; Noh, Y. Y., Organic light detectors: Photodiodes and phototransistors. Advanced Materials 2013, 25 (31), 4267-4295.

15. Koppens, F. H. L.; Mueller, T.; Avouris, P.; Ferrari, A. C.; Vitiello, M. S.; Polini, M., Photodetectors based on graphene, other two-dimensional materials and hybrid systems. Nature Nanotechnology 2014, 9 (10), 780-793. 
16. Wojciechowski, J. R.; Shriver-Lake, L. C.; Yamaguchi, M. Y.; Füreder, E.; Pieler, R.; Schamesberger, M.; Winder, C.; Prall, H. J.; Sonnleitner, M.; Ligler, F. S., Organic photodiodes for biosensor miniaturization. Analytical Chemistry 2009, 81 (9), 3455-3461.

17. Basiricò, L.; Ciavatti, A.; Cramer, T.; Cosseddu, P.; Bonfiglio, A.; Fraboni, B., Direct X-ray photoconversion in flexible organic thin film devices operated below $1 \mathrm{v}$. Nature Communications 2016, 7.

18. Konstantatos, G.; Sargent, E. H., Nanostructured materials for photon detection. Nature Nanotechnology 2010, 5 (6), 391-400.

19. Kang, B. J.; Lee, C. K.; Oh, J. H., All-inkjet-printed electrical components and circuit fabrication on a plastic substrate. Microelectronic Engineering 2012, 97, 251-254.

20. Piqué, A.; Chrisey, D. B., Direct-write Technologies for Rapid Prototyping Applications: Sensors, Electronics, and Integrated Power Sources. Academic: 2002.

21. Singh, M.; Haverinen, H. M.; Dhagat, P.; Jabbour, G. E., Inkjet printing-process and its applications. Advanced Materials 2010, 22 (6), 673-685.

22. Seifert, T.; Sowade, E.; Roscher, F.; Wiemer, M.; Gessner, T.; Baumann, R. R., Additive manufacturing technologies compared: Morphology of deposits of silver ink using inkjet and aerosol jet printing. Industrial and Engineering Chemistry Research 2015, 54 (2), 769-779.

23. Lilliu, S.; Böberl, M.; Sramek, M.; Tedde, S. F.; Macdonald, J. E.; Hayden, O., Inkjet-printed organic photodiodes. Thin Solid Films 2011, 520 (1), 610-615.

24. Falco, A.; Cinà, L.; Scarpa, G.; Lugli, P.; Abdellah, A., Fully-sprayed and flexible organic photodiodes with transparent carbon nanotube electrodes. ACS Applied Materials and Interfaces 2014, 6 (13), 10593-10601.

25. Tedde, S. F.; Kern, J.; Sterzl, T.; Fürst, J.; Lugli, P.; Hayden, O., Fully spray coated organic photodiodes. Nano Letters 2009, 9 (3), 980-983.

26. Pierre, A.; Arias, A. C. In High-detectivity printed organic photodiodes for large area flexible imagers, Technical Digest - International Electron Devices Meeting, IEDM, 2017; pp 32.4.1-32.4.4.

27. Correia, V.; Mitra, K. Y.; Castro, H.; Rocha, J. G.; Sowade, E.; Baumann, R. R.; Lanceros-Mendez, S., Design and fabrication of multilayer inkjet-printed passive components for printed electronics circuit development. Journal of Manufacturing Processes 2018, 31, 364-371.

28. Batool, S.; Gill, R.; Arshad, M.; Siddiqi, H. M.; Qureshi, S. S., Layer-by-layer fabrication of nacre inspired epoxy/MMT multilayered composites. Journal of Applied Polymer Science 2018, 135 (14).

29. Büchele, P.; Richter, M.; Tedde, S. F.; Matt, G. J.; Ankah, G. N.; Fischer, R.; Biele, M.; Metzger, W.; Lilliu, S.; Bikondoa, O.; Macdonald, J. E.; Brabec, C. J.; Kraus, T.; Lemmer, U.; Schmidt, O., X-ray imaging with scintillatorsensitized hybrid organic photodetectors. Nature Photonics 2015, 9 (12), 843-848.

30. Krebs, F. C.; Gevorgyan, S. A.; Gholamkhass, B.; Holdcroft, S.; Schlenker, C.; Thompson, M. E.; Thompson, B. C.; Olson, D.; Ginley, D. S.; Shaheen, S. E.; Alshareef, H. N.; Murphy, J. W.; Youngblood, W. J.; Heston, N. C.; Reynolds, J. R.; Jia, S.; Laird, D.; Tuladhar, S. M.; Dane, J. G. A.; Atienzar, P.; Nelson, J.; Kroon, J. M.; Wienk, M. M.; Janssen, R. A. J.; Tvingstedt, K.; Zhang, F.; Andersson, M.; Inganäs, O.; Lira-Cantu, M.; de Bettignies, R.; Guillerez, S.; Aernouts, T.; Cheyns, D.; Lutsen, L.; Zimmermann, B.; Würfel, U.; Niggemann, M.; Schleiermacher, H. F.; Liska, P.; Grätzel, M.; Lianos, P.; Katz, E. A.; Lohwasser, W.; Jannon, B., A round robin study of flexible large-area roll-to-roll processed polymer solar cell modules. Solar Energy Materials and Solar Cells 2009, 93 (11), 1968-1977.

31. Sahu, S.; Pal, A. J. In Photodetectors based on organic semiconductors: Optimization of device architectures, Proceedings of the 14th International Workshop on the Physics of Semiconductor Devices, IWPSD, 2007; pp 560562.

32. Pierre, A.; Deckman, I.; Lechêne, P. B.; Arias, A. C., High Detectivity All-Printed Organic Photodiodes. Advanced Materials 2015, 27 (41), 6411-6417.

33. Rocha, J. G.; Dias, R. A.; Goncalves, L.; Minas, G.; Ferreira, A.; Costa, C. M.; Lanceros-Mendez, S., X-ray image detector based on light guides and scintillators. IEEE Sensors Journal 2009, 9 (9), 1154-1159.

34. Oliveira, J.; Martins, P. M.; Martins, P.; Correia, V.; Rocha, J. G.; Lanceros-Mendez, S., Increasing X-ray to visible transduction performance of Gd203:Eu3+PVDF composites by PPO/POPOP addition. Composites Part B: Engineering 2016, 91, 610-614.

35. Oliveira, J.; Martins, P. M.; Martins, P.; Correia, V.; Rocha, J. G.; Lanceros-Mendez, S., Gd203:Eu3+/PPO/POPOP/PS composites for digital imaging radiation detectors. Applied Physics A: Materials Science and Processing 2015.

36. Oliveira, J.; Correia, V.; Costa, P.; Francesko, A.; Rocha, G.; Lanceros-Mendez, S., Stretchable scintillator composites for indirect X-ray detectors. Composites Part B: Engineering 2018, 133, 226-231.

37. $\quad$ Kang, Z.; Zhang, Y.; Menkara, H.; Wagner, B. K.; Summers, C. J.; Lawrence, W.; Nagarkar, V., CdTe quantum dots and polymer nanocomposites for x-ray scintillation and imaging. Applied Physics Letters 2011, 98 (18).

38. Boucher, R. A.; Bauch, J.; Wünsche, D.; Lackner, G.; Majumder, A., A carbon nanotube based x-ray detector. Nanotechnology 2016, 27 (47).

39. Paik, T.; Gordon, T. R.; Prantner, A. M.; Yun, H.; Murray, C. B., Designing tripodal and triangular gadolinium oxide nanoplates and self-assembled nanofibrils as potential multimodal bioimaging probes. ACS Nano 2013, 7 (3), 2850-2859.

40. $\quad$ Cai, W.; Chen, Q.; Cherepy, N.; Dooraghi, A.; Kishpaugh, D.; Chatziioannou, A.; Payne, S.; Xiang, W.; Pei, Q., Synthesis of bulk-size transparent gadolinium oxide-polymer nanocomposites for gamma ray spectroscopy. Journal of Materials Chemistry C 2013, 1 (10), 1970-1976. 
41. Gu, M.; Zhou, F.; Liu, X. L.; Ni, C.; Huang, S. M.; Liu, B., Preparation and luminescence properties of transparent Gd203:Eu thick film. Guang Pu Xue Yu Guang Pu Fen Xi/Spectroscopy and Spectral Analysis 2010, 30 (3), 603-606.

42. Sirringhaus, H.; Tessler, N.; Friend, R. H., Integrated optoelectronic devices based on conjugated polymers. Science 1998, 280 (5370), 1741-1744.

43. Kanagasekaran, T.; Shimotani, H.; Shimizu, R.; Hitosugi, T.; Tanigaki, K., A new electrode design for ambipolar injection in organic semiconductors. Nature Communications 2017, 8 (1).

44. Yamane, H.; Kanai, K.; Ouchi, Y.; Ueno, N.; Seki, K., Impact of interface geometric structure on organicmetal interface energetics and subsequent films electronic structure. Journal of Electron Spectroscopy and Related Phenomena 2009, 174 (1-3), 28-34.

45. Koch, N.; Heimel, G.; Wu, J.; Zojer, E.; Johnson, R. L.; Brédas, J. L.; Müllen, K.; Rabe, J. P., Influence of molecular conformation on organic/metal interface energetics. Chemical Physics Letters 2005, 413 (4-6), 390-395. 46. Furukawa, Y., Electronic absorption and vibrational spectroscopies of conjugated conducting polymers. Journal of Physical Chemistry 1996, 100 (39), 15644-15653.

47. $\quad$ Antonuk, L. E.; Jee, K. W.; El-Mohri, Y.; Maolinbay, M.; Nassif, S.; Rong, X.; Zhao, Q.; Siewerdsen, J. H.; Street, R. A.; Shah, K. S., Strategies to improve the signal and noise performance of active matrix, flat-panel imagers for diagnostic X-ray applications. Medical Physics 2000, 27 (2), 289-306.

48. Nikl, M., Scintillation detectors for x-rays. Measurement Science and Technology 2006, 17 (4), R37-R54.

49. Coling, D.; Kachar, B., Principles and application of fluorescence microscopy. Current protocols in molecular biology / edited by Frederick M. Ausubel ... [et al.] 2001, Chapter 14.

50. Ploem, J. S.; Tanke, H. J., Introduction to fluorescence microscopy. Oxford University Press, Incorporated: 1987.

51. Derenzo, S. E.; Weber, M. J.; Bourret-Courchesne, E.; Klintenberg, M. K., The quest for the ideal inorganic scintillator. Nuclear Instruments and Methods in Physics Research, Section A: Accelerators, Spectrometers, Detectors and Associated Equipment 2003, 505 (1-2), 111-117.

52. Kubitscheck, U., Fluorescence Microscopy: From Principles to Biological Applications. 2013.

53. Li, Y.; Lan, L.; Sun, S.; Lin, Z.; Gao, P.; Song, W.; Song, E.; Zhang, P.; Peng, J., All Inkjet-Printed Metal-Oxide Thin-Film Transistor Array with Good Stability and Uniformity Using Surface-Energy Patterns. ACS Applied Materials and Interfaces 2017, 9 (9), 8194-8200.

54. Sowade, E.; Mitra, K. Y.; Ramon, E.; Martinez-Domingo, C.; Villani, F.; Loffredo, F.; Gomes, H. L.; Baumann, R. R., Up-scaling of the manufacturing of all-inkjet-printed organic thin-film transistors: Device performance and manufacturing yield of transistor arrays. Organic Electronics: physics, materials, applications 2016, 30, 237-246. 55. Martins, P. M.; Martins, P.; Correia, V.; Rocha, J. G.; Lanceros-Mendez, S., Gd203:Eu Nanoparticle-Based Poly(vinylidene fluoride) Composites for Indirect X-ray Detection. Journal of Electronic Materials 2014, 44 (1), 129-135.

56. Cha, B. K.; Kim, J. Y.; Kim, T. J.; Sim, C.; Cho, G., Fabrication and imaging characterization of high sensitive $\mathrm{CsI}(\mathrm{Tl})$ and $\mathrm{Gd} 2 \mathrm{O} 2 \mathrm{~S}(\mathrm{~Tb})$ scintillator screens for X-ray imaging detectors. Radiation Measurements 2010, 45 (3-6), 742-745.

57. Kalyvas, N.; Liaparinos, P.; Michail, C.; David, S.; Fountos, G.; Wójtowicz, M.; Zych, E.; Kandarakis, I., Studying the luminescence efficiency of Lu 20 3:Eu nanophosphor material for digital X-ray imaging applications. Applied Physics A: Materials Science and Processing 2012, 106 (1), 131-136.

58. $\quad$ Seferis, I.; Michail, C.; Valais, I.; Zeler, J.; Liaparinos, P.; Fountos, G.; Kalyvas, N.; David, S.; Stromatia, F.; Zych, E.; Kandarakis, I.; Panayiotakis, G., Light emission efficiency and imaging performance of Lu20 3:Eu nanophosphor under X-ray radiography conditions: Comparison with Gd2O2S:Eu. Journal of Luminescence 2014, 151, 229-234.

59. Starkenburg, D. J.; Johns, P. M.; Baciak, J. E.; Nino, J. C.; Xue, J., Thin film organic photodetectors for indirect $\mathrm{X}$-ray detection demonstrating low dose rate sensitivity at low voltage operation. Journal of Applied Physics 2017, 122 (22).

\section{Table of Contents}




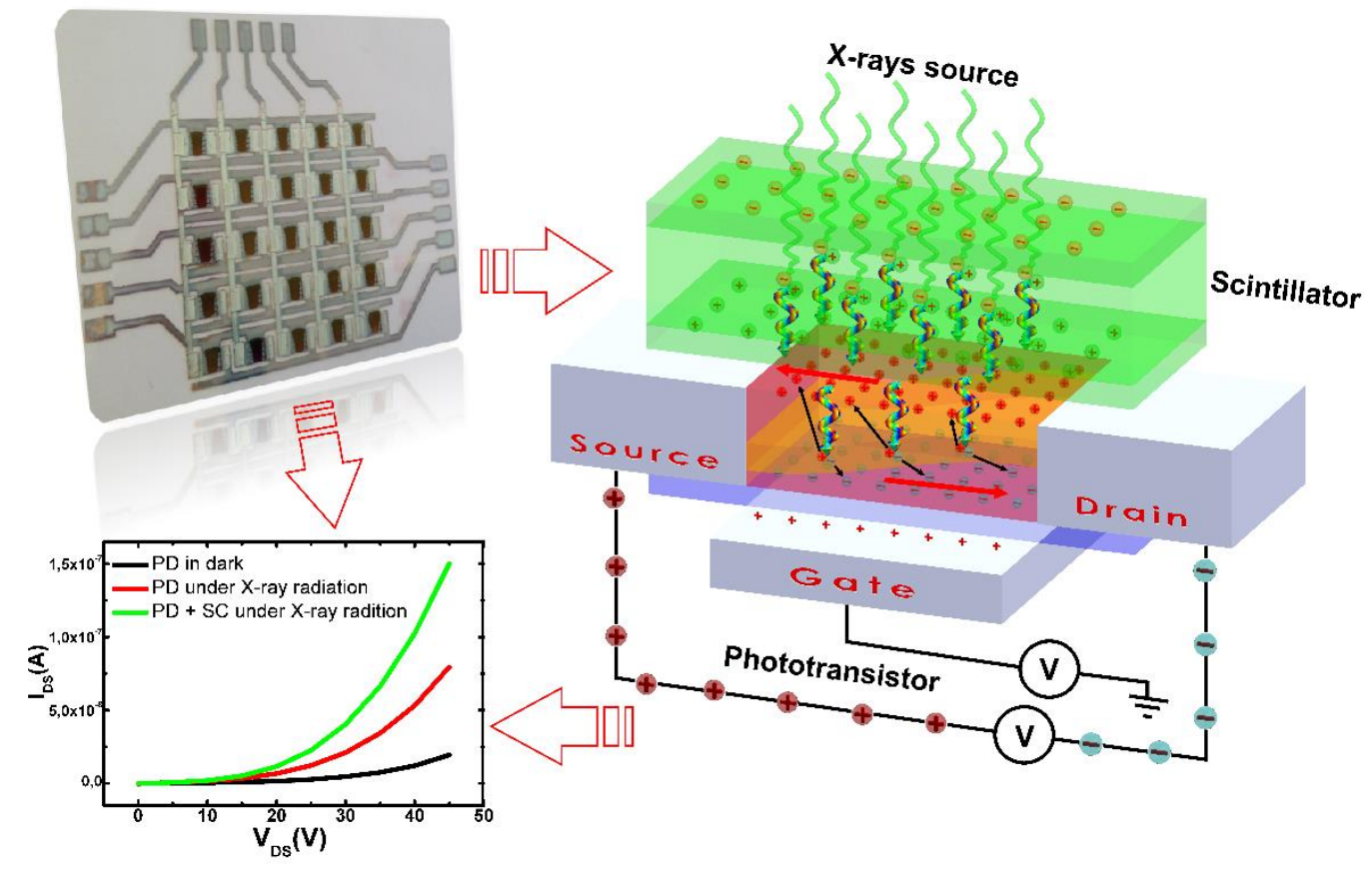

